Case Study

\title{
Composition and Distribution of Benthic Diatoms in Different Habitats of Burdur River Basin
}

\section{Burdur Nehir Havzasındaki Farklı Habitatlarda Bentik Diyatome Kompozisyonu ve Dağılımı}

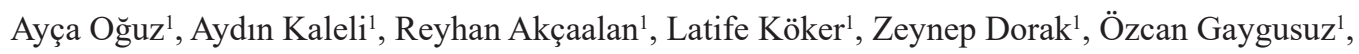 \\ Fatih Aydın', Tolga Çetin², Yakup Karaaslan' ${ }^{2}$, Meriç Albay ${ }^{1}$ \\ ${ }^{1}$ Department of Freshwater Resource and Management, Faculty of Aquatic Sciences, \\ Istanbul University \\ ayca.oguzcam@istanbul.edu.tr (https://orcid.org/0000-0002-0711-2967), aydin.kaleli@istanbul.edu. \\ tr (https://orcid.org/0000-0003-3843-1335), akcaalan@istanbul.edu.tr ( https://orcid.org/0000-0002- \\ 0756-8972), latife.koker@istanbul.edu.tr (https://orcid.org/0000-0002-9134-2801),zdorak@istanbul. \\ edu.tr (https://orcid.org/0000-0003-4782-3082), ozcan.gaygusuz@istanbul.edu.tr ( https://orcid. \\ org/0000-0001-6861-6221), fatih.aydin@istanbul.edu.tr (https://orcid.org/0000-0002-5003-2620), \\ merbay@istanbul.edu.tr (https://orcid.org/0000-0001-9726-945X) \\ ${ }^{2}$ Ministry of Agriculture and Forestry, General Directorate of Water Management \\ tolga.cetin@tarimorman.gov.tr (https://orcid.org/0000-0002-7817-3222),yakup.karaaslan@ \\ tarimorman.gov.tr (https://orcid.org/0000-0001-8993-4771) \\ Received Date: 11.12.2019, Accepted Date: 23.12.2019
}

\begin{abstract}
Diatoms constitute an essential component for biomonitoring studies to determine the ecological quality of waterbodies. In this study, benthic diatoms of Burdur River Basin were investigated as a consequence of a project on river basin management plan. This is the first detailed taxonomical study of diatoms taken from 13 streams, 4 lakes and 6 reservoirs of Burdur River Basin and a total of 223 taxa have been observed. Among genera, Navicula Bory (27) and Nitzschia Hassal (27) were represented with the highest numbers of taxa and followed by Gomphonema Agardh with a total of 22 species. Navicula antonii Lange-Bertalot and Nitzschia palea var. debilis (Kützing) Grunow had the highest relative abundance with $17.1 \%$ and $15.5 \%$ respectively. As a closed basin, salinity varied greatly from fresh to saline water between the sampling stations and diatom composition contained species with a different tolerance level. Navicula antonii and Nitzschia frustulum (Kützing) Grunow observed in all habitats indicating their euryhaline characters; however Navicula digitoconvergens was only detected in saline Acıgöl lake with a high relative abundance (38\%) in autumn. Other two dominant species were Halamphora coffeiformis (Kützing) Levkov and Navicula cincta (Ehrenberg) Ralfs in Acıgöl lake showing their tolerance to high salt content as a brackish species. According to first results, 11 species were new records for Turkish diatom flora. The high biodiversity of diatoms revealed the presence of different habitat characteristics within the basin. These results are an important contribution of Turkish diatom flora and could be useful for monitoring specific areas like Burdur River Basin.
\end{abstract}

Keywords: Burdur River Basin, diatoms, phytobenthos, salinity tolerance 


\section{$\ddot{O} z$}

Diyatomeler, su kütlelerinin ekolojik kalitesini belirlemek için yapılan biyolojik izleme çalışmalarında kullanılan önemli bir bileşendir. Bu çalışmada, Burdur Nehir Havzası'nın tatlı su bentik diyatomeleri nehir havzası yönetim planının hazırlanması projesi kapsamında 13 nehir, 4 göl ve 6 rezervuar olmak üzere toplam 23 noktadan örneklenmiştir. Çalışma kapsamında havzada bentik diyatomelerin ilk defa detaylı taksonomik incelenmesi yapılmış olup, toplamda 223 takson gözlenmiştir. Cinsler arasında Navicula Bory (27) ve Nitzschia Hassal (27) en fazla taksonla temsil edilmiş olup, bunu 22 tür ile Gomphonema Agardh takip etmiştir. Navicula antonii Lange-Bertalot ve Nitzschia palea var. debilis (Kützing) Grunow, sirasıyla \%17,1 and \%15,5 nisbi bolluk değerleri ile en yüksek nispi bolluğa sahip türler olmuştur. Kapalı bir havza olan Burdur Havzası'nda örnekleme alanlarının tuzluluk değerleri tatlı sudan tuzlu suya kadar farklılık göstermiş olup, diyatome kompozisyonu da farklı tuzluluk tolerans seviyelerine sahip türleri içermektedir. Örihalin karaktere sahip olan Navicula antonii ve Nitzschia frustulum bütün habitatlarda gözlenirken, Navicula digitoconvergens sadece tuzlu su karakterine sahip olan Acıgöl'de yüksek nisbi bolluğa (\%38) ulaşmıştır. Acıgöl'de tespit edilen diğer iki dominant tür olan Halamphora coffeiformis ve Navicula cincta türleri de acısu karakterindeki sularda artı̧̧ yaptı̆̆ 1 bilinen türlerdir ve bu havzada yüksek tuzluluğa tolerans göstermişlerdir. Elde edilen ilk sonuçlara göre, 11 tür Türkiye diyatome florası için yeni kayıtlardır. Diyatomelerin yüksek biyoçeşitliliği Burdur Nehir Havzası'ndaki farklı habitat karakteristiklerinin varlığını ortaya koymuştur. Bu sonuçlar, Türkiye diyatome florasına önemli bir katkı sağlayacak ve Burdur Nehir Havzası gibi özel alanların izlenmesinde yararlı olacaktır.

Anahtar kelimeler: Burdur Nehir Havzasl, diyatomeler, fitobentoz, tuzluluk toleransı

\section{Introduction}

Amongst the algae groups, diatoms have been a subject of study, especially in recent years due to the biomonitoring of freshwater bodies for water quality assessments. Their prompt response to the environmental changes and their presence throughout the year together with rapid reproduction stages made diatoms an essential tool for monitoring freshwater systems. Studies were conducted in various waterbodies of Turkey to detect the environmental quality of the lakes (Dalkıran et al. 2016; Şanal \& Demir, 2018) and rivers (Atıc1, 1997; Karacaoğlu \& Dalkıran, 2017; Demir et al. 2017; Solak et al. 2018; Çelekli et al. 2019).

Burdur River Basin is located in the southwest of central Anatolia, and it is one of the smallest basins. In the neighbouring river basins, benthic diatoms had become an interest for the researchers to determine the flora of several streams or lakes. Çiçek \& Yamuç (2017) investigated epilithic algae in relation to environmental factors in Kovada Lake in Antalya River Basin. In Büyük Menderes, there were many studies concerning benthic diatoms as well; e.g., Barlas et al. (2002), studied the epilithic algae of Akçapınar Stream and Kadın Azmağı Stream. 
Detection of ecological quality of rivers and lakes has become more important for the last decades in Europe, according to Water Framework Directive (WFD) regulations (Ács et al. 2004; Rimet, 2012). Similarly, studies were conducted to use biological components, including benthic diatoms, of aquatic ecosystems to assess the ecological quality based on WFD. As a result, basin-scale studies on diatom biodiversity and ecology have increased recently. Demir et al. (2017) reported diatom composition of Lake Eber and the streams in Akarçay River Basin, while Solak et al. (2018) analysed the distribution of diatoms in streams and reservoirs of Küçük Menderes River. Benthic diatom community of streams, lakes and reservoirs of Gediz River Basin was also studied by Solak et al. (2019). Unlike the other 24 river basins in Turkey, there is no data on benthic diatom composition in the lakes and streams of Burdur River Basin.

The aim of this research is to determine the benthic diatom flora of streams and lakes/reservoirs of Burdur River Basin and provide a taxonomical data for the environmental quality monitoring of the basin according to regulations of WFD (2000/60/EC).

\section{Method}

\section{Sampling}

Burdur River Basin is located in the southwest of Turkey, covering some natural lakes and wetlands such as Burdur Lake, Acıgöl Lake and Salda Lake (Figure 1). Sampling was carried out in 23 waterbodies including the streams, lakes and reservoirs. A total of 30 sampling points were selected in the basin area; however, sampling could not be performed due to the drought in seven locations (Table 1). Benthic diatom samples were taken twice a year (April and October 2018). According to Communique on Biological Monitoring (T.C. Resmi Gazete, 2019), one sample collected from lakes and reservoirs smaller than 50 ha; 2 samples in areas between 50-500 ha area and 3 samples from the lakes and reservoirs greater than 500 ha were taken. However, all samples were mixed and one subsample was prepared for each lake and reservoir. Epilithic diatom samples were collected from the submerged stones, and epiphytic samples were collected from macrophytes. Physicochemical measurements were performed monthly in 2018 . 


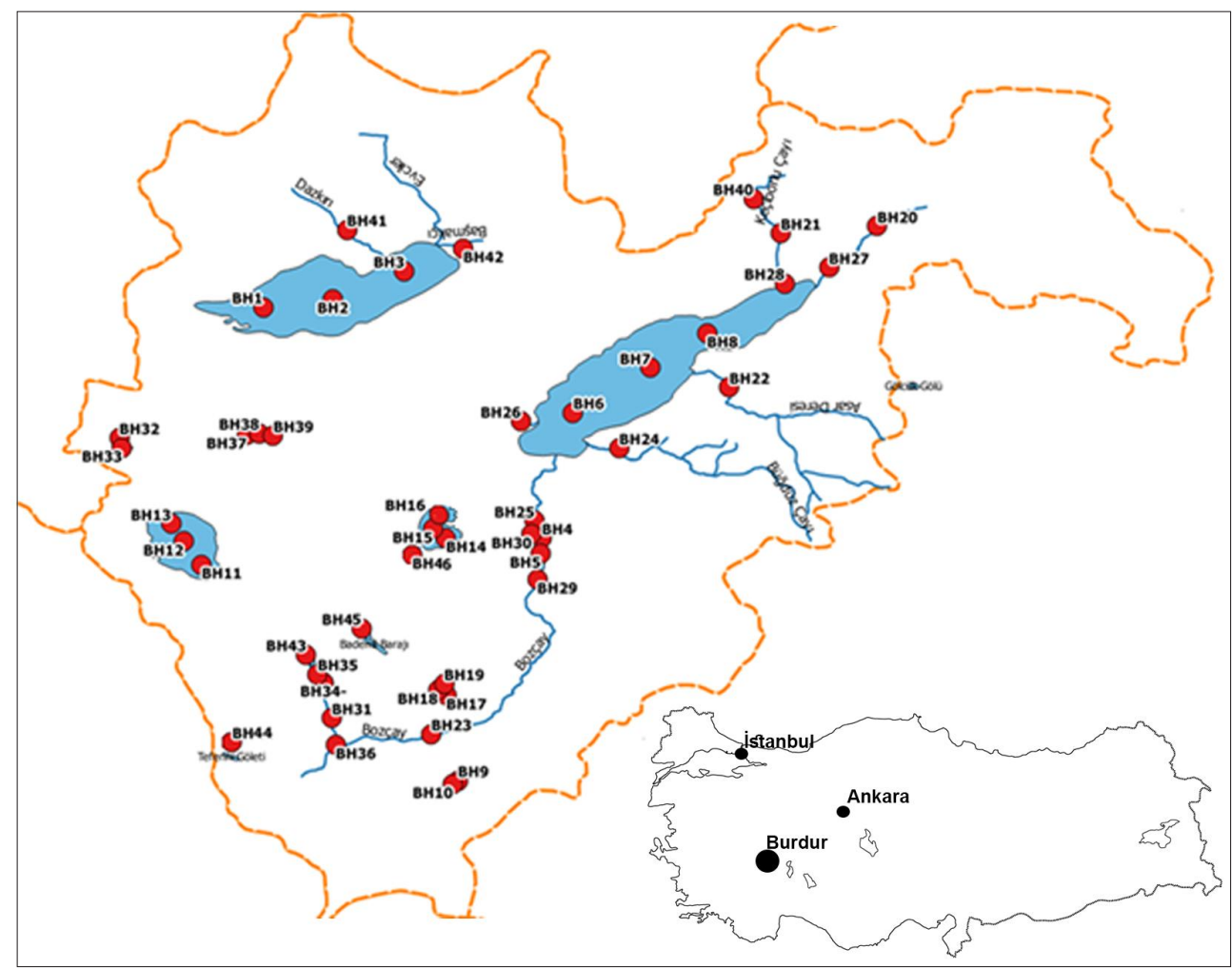

Figure 1. Study area and sampling stations.

\section{Diatom Analysis}

Samples were cleaned from organic material by boiling with $\mathrm{H}_{2} \mathrm{O}_{2}$ and washed by distilled water several times. In order to remove the carbonates, samples were treated with $10 \% \mathrm{HCl}$. Frustules were air-dried and mounted in Naphrax ${ }^{\circledR}$. Zeiss Axio Observer Z1 (Carl Zeiss microscopy Gmbh, Jena, Germany) microscope was used for light microscopy (LM) observations at Limnology Laboratory, Department of Freshwater Resource and Management, Istanbul University.

Diatom valves were identified according to the following literature; Krammer \& Lange-Bertalot (1986, 1988, 1991a,b), Hofmann et al. (2011) and Kulikovskiy et al. (2016). Taxonomic classification and nomenclatural on genera and taxa names follow the latest updates from Guiry \& Guiry (2019) and Kociolek et al. (2019). Slides and processed materials were deposited at the collection of the Department of Freshwater Resource and Management, Istanbul University and the Ministry of Agriculture and Forestry archives. 
The relative abundance of the species was expressed as percentages of the total number of frustules counted. The relative abundance (RA) of particular taxa and the taxa richness of the assemblages were estimated on the basis of at least 300 diatom valves counted per sample. The RA of the species identified in lakes, reservoirs and streams were determined separately and the species constituting higher than $5 \%$ were evaluated in Table 4.

Table 1

\section{Burdur River Basin Sampling Coordinates}

\begin{tabular}{|c|c|c|c|c|c|c|}
\hline \multirow{2}{*}{ Code } & \multirow{2}{*}{ Name } & \multirow{2}{*}{ Category } & \multirow{2}{*}{ Salinity } & \multirow{2}{*}{ Province } & \multicolumn{2}{|c|}{ Coordinates } \\
\hline & & & & & $\mathrm{X}$ & $\mathrm{Y}$ \\
\hline BH1 & \multirow{3}{*}{ Acıgöl } & \multirow{3}{*}{ Lake } & \multirow{3}{*}{$\begin{array}{l}\text { Saline } \\
(39 \%)\end{array}$} & \multirow{3}{*}{ Afyon } & 37.81844 & 29.77163 \\
\hline $\mathrm{BH} 2$ & & & & & 37.82721 & 29.84914 \\
\hline BH3 & & & & & 37.85838 & 29.92875 \\
\hline $\mathrm{BH} 4$ & \multirow{2}{*}{ Karaçal } & \multirow{2}{*}{ Reservoir } & \multirow{2}{*}{ Freshwater } & \multirow{2}{*}{ Burdur } & 37.56175 & 30.08104 \\
\hline BH5 & & & & & 37.54444 & 30.08007 \\
\hline BH6 & \multirow{3}{*}{ Burdur } & \multirow{3}{*}{ Lake } & \multirow{3}{*}{$\begin{array}{l}\text { Brackish } \\
(18.2 \% \text { ) }\end{array}$} & \multirow{3}{*}{ Burdur } & 37.70125 & 30.11597 \\
\hline $\mathrm{BH} 7$ & & & & & 37.75088 & 30.20211 \\
\hline BH8 & & & & & 37.78928 & 30.26449 \\
\hline BH9 & \multirow{2}{*}{ Belenli } & \multirow{2}{*}{ Reservoir } & \multirow{2}{*}{ Freshwater } & \multirow{2}{*}{ Burdur } & 37.29235 & 29.98792 \\
\hline BH10 & & & & & 37.28849 & 29.98258 \\
\hline BH11 & \multirow{3}{*}{ Salda } & \multirow{3}{*}{ Lake } & \multirow{3}{*}{$\begin{array}{c}\text { Brackish } \\
(1.1 \% 0)\end{array}$} & \multirow{3}{*}{ Burdur } & 37.53238 & 29.70349 \\
\hline BH12 & & & & & 37.55788 & 29.68351 \\
\hline $\mathrm{BH} 13$ & & & & & 37.57749 & 29.66961 \\
\hline BH14 & \multirow{3}{*}{ Yarışlı } & \multirow{3}{*}{$\begin{array}{l}\text { Lake } \\
\text { (Dry) }\end{array}$} & \multirow{3}{*}{-} & \multirow{3}{*}{ Burdur } & 37.56182 & 29.97482 \\
\hline BH15 & & & & & 37.57202 & 29.96020 \\
\hline BH16 & & & & & 37.58831 & 29.96729 \\
\hline BH17 & \multirow{3}{*}{ Karataş } & \multirow{3}{*}{ Lake } & \multirow{3}{*}{ Freshwater } & & 37.38701 & 29.97508 \\
\hline BH18 & & & & Burdur & 37.39292 & 29.96579 \\
\hline BH19 & & & & & 37.39989 & 29.97259 \\
\hline $\mathrm{BH} 20$ & Gönen & $\begin{array}{l}\text { Stream } \\
\text { (Dry in } \\
\text { October) }\end{array}$ & Freshwater & Isparta & 37.90820 & 30.45358 \\
\hline $\mathrm{BH} 21$ & Çukurharman & Stream (Dry) & - & Isparta & 37.90046 & 30.34642 \\
\hline $\mathrm{BH} 22$ & Asar & Stream & $\begin{array}{l}\text { Brackish } \\
(0.53 \% 0)\end{array}$ & Burdur & 37.72920 & 30.28935 \\
\hline $\mathrm{BH} 23$ & Bozçay & Stream & Freshwater & Burdur & 37.34401 & 29.95839 \\
\hline
\end{tabular}




\begin{tabular}{|c|c|c|c|c|c|c|}
\hline \multirow{2}{*}{ Code } & \multirow{2}{*}{ Name } & \multirow{2}{*}{ Category } & \multirow{2}{*}{ Salinity } & \multirow{2}{*}{ Province } & \multicolumn{2}{|c|}{ Coordinates } \\
\hline & & & & & $\mathrm{X}$ & $\mathrm{Y}$ \\
\hline $\mathrm{BH} 24$ & Büğgüz & $\begin{array}{l}\text { Stream } \\
\text { (Dry in } \\
\text { October) }\end{array}$ & Freshwater & Burdur & 37.66174 & 30.16703 \\
\hline $\mathrm{BH} 25$ & Bozçay & Stream & Freshwater & Burdur & 37.58073 & 30.07316 \\
\hline BH26 & Ulupınar & Stream (Dry) & - & Burdur & 37.69160 & 30.05794 \\
\hline BH27 & Gönen & Stream & $\begin{array}{c}\text { Brackish } \\
(1.9 \% 0) \\
\end{array}$ & Isparta & 37.86231 & 30.39985 \\
\hline $\mathrm{BH} 28$ & Çukurharman & Stream & $\begin{array}{c}\text { Brackish } \\
(0.7 \% \text { o })\end{array}$ & Isparta & 37.84485 & 30.35068 \\
\hline BH29 & Bozçay & Stream & Freshwater & Burdur & 37.51586 & 30.07599 \\
\hline BH30 & Bozçay & Stream & Freshwater & Burdur & 37.56599 & 30.07000 \\
\hline BH31 & Karamanlı & Stream (Dry) & - & Burdur & 37.36173 & 29.84796 \\
\hline BH32 & \multirow{2}{*}{ Beylerli } & \multirow{2}{*}{ Reservoir } & \multirow{2}{*}{ Freshwater } & \multirow{2}{*}{ Denizli } & 37.67316 & 29.61199 \\
\hline BH33 & & & & & 37.66217 & 29.61534 \\
\hline $\mathrm{BH} 34$ & \multirow{2}{*}{ Karamanlı } & \multirow{2}{*}{ Reservoir } & \multirow{2}{*}{ Freshwater } & \multirow{2}{*}{ Burdur } & 37.40121 & 29.83880 \\
\hline BH35 & & & & & 37.41066 & 29.83226 \\
\hline BH36 & Sar1 & Stream (Dry) & - & Burdur & 37.33204 & 29.85228 \\
\hline BH37 & \multirow{3}{*}{ Akgöl } & \multirow{3}{*}{$\begin{array}{l}\text { Lake } \\
\text { (Dry) }\end{array}$} & \multirow{3}{*}{-} & \multirow{3}{*}{ Burdur } & 37.67674 & 29.75361 \\
\hline BH38 & & & & & 37.67821 & 29.76745 \\
\hline BH39 & & & & & 37.67559 & 29.78304 \\
\hline BH40 & Keçiborlu & $\begin{array}{c}\text { Stream } \\
\text { (Dry in April) }\end{array}$ & $\begin{array}{l}\text { Brackish } \\
(1.45 \% 0)\end{array}$ & Isparta & 37.93891 & 30.31606 \\
\hline BH41 & Dazkırı & Stream (Dry) & - & Afyon & 37.90446 & 29.86472 \\
\hline $\mathrm{BH} 42$ & Başmakçı & $\begin{array}{c}\text { Stream } \\
\text { (Dry in April) }\end{array}$ & $\begin{array}{c}\text { Brackish } \\
(6 \%)\end{array}$ & Afyon & 37.88374 & 29.99386 \\
\hline $\mathrm{BH} 43$ & Özdere & Stream & Freshwater & Burdur & 37.43224 & 29.81980 \\
\hline BH44 & Tefenni & Reservoir & Freshwater & Burdur & 37.31703 & 29.74366 \\
\hline $\mathrm{BH} 45$ & Bademli & Reservoir & Freshwater & Burdur & 37.43503 & 29.90621 \\
\hline $\mathrm{BH} 46$ & Yarışlı & Stream & Freshwater & Burdur & 37.43503 & 29.90621 \\
\hline
\end{tabular}




\section{Results}

\section{Physicochemical Parameters}

The physicochemical measurements revealed that reservoirs, lakes and streams in the basin were generally alkaline. The mean conductivity values were generally low in reservoirs and streams $\left(0.5\right.$ and $1.7 \mathrm{mS} \mathrm{cm}^{-1}$, respectively) but higher in lakes $\left(22.3 \mathrm{mS} \mathrm{cm}^{-1}\right)$. Notably, in Burdur and Acıgöl lakes, conductivity was higher throughout the year, and the mean conductivity values were $29.4 \mathrm{mS} \mathrm{cm}^{-1}$ and 57.2 $\mathrm{mS} \mathrm{cm} \mathrm{cm}^{-1}$ respectively. Significant changes were observed in dissolved oxygen values in reservoirs, lakes and streams throughout the year. Although the lowest dissolved oxygen (DO) values were observed in Burdur Lake as $2.7 \mathrm{mg} \mathrm{L}^{-1}$ and Acıgöl Lake as $1 \mathrm{mg} \mathrm{L}^{-1}$, the average values were between 6.9 and $7.8 \mathrm{mg} \mathrm{L}^{-1}$ in the reservoirs and lakes (Table 2).

As a closed basin, salinity varied remarkably between freshwater to saline among studied areas. While all reservoirs were classified as freshwater $(<0.5 \%)$, streams were categorized between fresh to brackish water $(<0.5-6 \%$ ). On the other hand, salinity variation was higher in lakes, from freshwater (Karataş Lake, $<0.5 \%$ ) to brackish (Salda Lake, 1\%o and Burdur Lake, 18\%) and even saline environment (Acıgöl Lake, 39 \%o).

Table 2

Mean, Min and Max Values of Selected Water Quality Parameters Measured Studied Areas

\begin{tabular}{cccccccccccccccccc}
\hline & $\begin{array}{c}\text { Temperature } \\
\left({ }^{\circ} \mathrm{C}\right)\end{array}$ & & $\mathrm{pH}$ & \multicolumn{6}{c}{$\begin{array}{c}\text { Conductivity } \\
\left(\mathrm{mS} \mathrm{cm}^{-1}\right)\end{array}$} & $\begin{array}{c}\text { Dissolved Oxygen } \\
\left(\mathrm{mg} \mathrm{L}^{-1}\right)\end{array}$ & $\begin{array}{c}\text { Salinity } \\
(\%)\end{array}$ \\
& Mean & Min & Max & Mean & Min & Max & Mean & Min & Max & Mean Min & Max & Mean & Min & Max \\
\hline Reservoirs & 13.7 & 3.8 & 24.0 & 8.5 & 7.4 & 9.3 & 0.5 & 0.2 & 0.6 & 6.9 & 3.0 & 11.4 & $<0,5$ & $<0,5$ & $<0,5$ \\
Lakes & 15.2 & 5.1 & 29.0 & 8.8 & 6.4 & 9.6 & 22.3 & 0.5 & 69.6 & 7.8 & 1.0 & 11.4 & 19.4 & $<0,5$ & 47.6 \\
Streams & 15.8 & 2.2 & 27.9 & 8.3 & 7.0 & 9.7 & 1.7 & 0.3 & 12.9 & 6.9 & 0.5 & 12.7 & 2.7 & $<0,5$ & 7.5 \\
\hline
\end{tabular}




\section{Diatom Composition}

Composition and distribution of diatoms have been studied in two seasons, and a total of 223 taxa belonging to 57 genera were identified; within these taxa, 11 were identified in genera level. The seasonal composition of species were presented according to sampling habitats; streams, lakes and reservoirs (Table 3).

Table 3

Diatom Composition and Distribution in Streams, Lakes and Reservoirs of Burdur River Basin

\begin{tabular}{|c|c|c|c|c|c|c|}
\hline \multirow[t]{2}{*}{ Taxa } & \multicolumn{2}{|c|}{ Stream } & \multicolumn{2}{|c|}{ Lake } & \multicolumn{2}{|c|}{ Reservoir } \\
\hline & Sp. & Aut. & Sp. & Aut. & Sp. & Aut. \\
\hline Achnanthes petersenii Hustedt & + & & & & & \\
\hline Achnanthidium eutrophilum (Lange-Bertalot) Lange-Bertalot & + & & & & & + \\
\hline Achnanthidium minutissimum (Kützing) Czarnecki & + & + & & & + & \\
\hline $\begin{array}{l}\text { Achnanthidium minutissimum var. jackii (Rabenhorst) Lange- } \\
\text { Bertalot }\end{array}$ & + & + & + & + & + & + \\
\hline Adlafia minuscula var. muralis (Grunow) Lange-Bertalot & & + & & & & \\
\hline Amphora aequalis Krammer & & + & & & & \\
\hline Amphora alpestris Levkov & & + & & & & \\
\hline Amphora copulata (Kützing) Schoeman \& R.E.M. Archibald & & + & & & & + \\
\hline Amphora inariensis Krammer & & + & & & & + \\
\hline Amphora indistincta Levkov & & & & & & + \\
\hline Amphora lange-bertalotii Levkov \& Metzeltin & & & & & & + \\
\hline Amphora ovalis (Kützing) Kützing & & + & & & + & + \\
\hline Amphora pediculus (Kützing) Grunow & + & + & & & + & + \\
\hline Amphora stechlinensis Levkov \& Metzeltin & + & & & & & + \\
\hline Anomoeoneis sphaerophora Pfitzer & & + & & & & \\
\hline Aulacoseira ambigua (Grunow) Simonsen & & & & & + & + \\
\hline Aulacoseira italica (Ehrenberg) Simonsen & + & & & & & \\
\hline Berkeleya sp. & & + & + & + & & + \\
\hline Caloneis amphisbaena (Bory) Cleve & & + & & & & \\
\hline Caloneis bacillum (Grunow) Cleve & & & & & + & \\
\hline Caloneis silicula (Ehrenberg) Cleve & & & + & & & \\
\hline Cocconeis lineata Ehrenberg & + & + & & + & + & + \\
\hline
\end{tabular}




\begin{tabular}{|c|c|c|c|c|c|c|}
\hline \multirow[t]{2}{*}{ Taxa } & \multicolumn{2}{|c|}{ Stream } & \multicolumn{2}{|c|}{ Lake } & \multicolumn{2}{|c|}{ Reservoir } \\
\hline & Sp. & Aut. & Sp. & Aut. & Sp. & Aut. \\
\hline Cocconeis pediculus Ehrenberg & + & + & & & & \\
\hline Cocconeis placentula var. placentula Ehrenberg & + & + & & + & + & + \\
\hline Craticula accomoda (Hustedt) D.G. Mann & & & & & & + \\
\hline Craticula ambigua (Ehrenberg) D.G. Mann & + & + & & + & + & + \\
\hline Craticula buderi (Hustedt) Lange-Bertalot* & + & + & & & & \\
\hline Craticula cuspidata (Kützing) D.G. Mann & + & & & & & \\
\hline Craticula subminuscula (Manguin) Wetzel \& Ector & + & + & & & & \\
\hline Craticula sp. & & + & & & & \\
\hline Cyclostephanos dubius (Hustedt) Round & & + & & & & \\
\hline $\begin{array}{llll}\text { Cyclostephanos invisitatus } & \text { (Hohn\& Hellermann) Theriot, } \\
\text { Stoermer \& Håkasson } & & & \\
\end{array}$ & & & & & & + \\
\hline Cyclotella meneghiniana Kützing & + & + & & & + & \\
\hline Cymbella affinis Kützing & + & & & & & + \\
\hline Cymbella cymbiformis Agardh & + & + & + & + & + & + \\
\hline Cymbella dorsenotata Østrup & + & & & & & + \\
\hline Cymbella excisa Kützing & & + & & & & \\
\hline Cymbella helvetica Kützing & & & & & & + \\
\hline Cymbella lanceolata (Agardh) Agardh & & & + & & & \\
\hline Cymbella lange-bertalotii Krammer* & & + & & & + & + \\
\hline Cymbella neocistula Krammer & & & & & & + \\
\hline Cymbella vulgata Krammer & & + & & & & \\
\hline Cymbopleura amphicephala (Nägeli) Krammer & & + & + & & + & + \\
\hline Cymbopleura inaequalis (Ehrenberg) Krammer & & & & & & + \\
\hline Cymbopleura rhomboidea Krammer & & & & & & + \\
\hline Cymbellafalsa diluviana (Krasske) Lange-Bertalot \& Metzeltin & + & & & & & \\
\hline Diatoma moniliformis (Kützing) D.M. Williams & + & & & + & + & \\
\hline Diatoma tenuis C. Agardh & & + & & & & \\
\hline Diatoma vulgaris Bory & + & + & & & & \\
\hline Diploneis elliptica (Kützing) Cleve & & & & & + & \\
\hline Diploneis krammeri Lange-Bertalot \& E. Reichardt & + & & & & + & \\
\hline Diploneis parma Cleve & & + & & & & \\
\hline Dorofeyukea kotschyi Kulikovskiy et al. & & + & & & + & \\
\hline
\end{tabular}




\begin{tabular}{|c|c|c|c|c|c|c|}
\hline \multirow[t]{2}{*}{ Taxa } & \multicolumn{2}{|c|}{ Stream } & \multicolumn{2}{|c|}{ Lake } & \multicolumn{2}{|c|}{ Reservoir } \\
\hline & Sp. & Aut. & Sp. & Aut. & Sp. & Aut. \\
\hline Encyonema caespitosum Kützing & + & + & + & & + & + \\
\hline Encyonema hebridicum Grunow ex Cleve & & & & & & + \\
\hline Encyonema lacustre (C. Agardh) Pantocsek & & + & & & & + \\
\hline Encyonema lange-bertalotii Krammer & & + & & & & \\
\hline Encyonema latum Krammer & & + & & & & \\
\hline Encyonema leibleinii (C. Agardh) Silva et al. & & & + & & + & \\
\hline Encyonema minutum (Hilse) D.G. Mann & & + & & & + & + \\
\hline Encyonema silesiacum (Bleisch) D.G. Mann & & + & + & + & + & + \\
\hline Encyonema ventricosum (C. Agardh) Grunow & + & & & & + & + \\
\hline Encyonema vulgare Krammer* & & & & & + & + \\
\hline Encyonopsis cesatii (Rabenhorst) Krammer & & + & & & + & \\
\hline Encyonopsis microcephala (Grunow) Krammer & & + & & + & + & \\
\hline Encyonopsis minuta Krammer \& E. Reichardt & & & & + & + & \\
\hline Encyonopsis subminuta Krammer \& E. Reichardt & + & + & & + & + & + \\
\hline Encyonopsis sp. & & + & & & & \\
\hline Epithemia adnata (Kützing) Brébisson & & + & & & & \\
\hline Epithemia gibba (Ehrenberg) Kützing & & + & & & & + \\
\hline Epithemia smithii Carruthers & & & & + & & + \\
\hline Epithemia sorex Kützing & & + & & + & & \\
\hline Fallacia pygmaea (Kützing) Stickle \& D.G. Mann & + & + & + & + & + & \\
\hline Fragilaria gracilis Østrup & & & & & & + \\
\hline Fragilaria henryi Lange-Bertalot & & & & & + & \\
\hline Fragilaria pararumpens Lange-Bertalot, G. Hofmann \& Werum & & & & + & & \\
\hline Fragilaria radians (Kützing) D.M. Williams \& Round & + & + & & & & \\
\hline $\begin{array}{l}\text { Fragilaria tenera var. nanana (Lange-Bertalot) Lange-Bertalot } \\
\& \text { S. Ulrich }\end{array}$ & & + & & & & \\
\hline Fragilaria vaucheriae (Kützing) J.B. Petersen & + & & + & + & + & + \\
\hline Frustulia sp. & & & & & + & \\
\hline Geissleria decussis (Østrup) Lange-Bertalot \& Metzeltin & & & & & & + \\
\hline Gomphonema auritum A. Braun ex Kützing* & & & & & + & \\
\hline Gomphonema calcareum Cleve & & & & + & & \\
\hline Gomphonema clavatum Ehrenberg & + & + & & & & \\
\hline
\end{tabular}




\begin{tabular}{|c|c|c|c|c|c|c|}
\hline \multirow{2}{*}{ Taxa } & \multicolumn{2}{|c|}{ Stream } & \multicolumn{2}{|c|}{ Lake } & \multicolumn{2}{|c|}{ Reservoir } \\
\hline & Sp. & Aut. & Sp. & Aut. & Sp. & Aut. \\
\hline Gomphonema drutelingense E. Reichardt* & + & & & & & \\
\hline $\begin{array}{l}\text { Gomphonema exilissimum (Grunow) Lange-Bertalot \& E. } \\
\text { Reichardt* }\end{array}$ & & + & & & & + \\
\hline Gomphonema innocens E. Reichardt & + & & & & & \\
\hline Gomphonema italicum Kützing & & + & & & & + \\
\hline Gomphonema lippertii E. Reichardt \& Lange-Bertalot & & + & & & & \\
\hline Gomphonema minusculum Krasske & & + & & & & \\
\hline Gomphonema minutum (C. Agardh) C. Agardh & & + & & & & \\
\hline Gomphonema olivaceum (Hornemann) Brébisson & + & + & + & + & + & + \\
\hline Gomphonema pala E. Reichardt & & + & & & & \\
\hline Gomphonema parvulum (Kützing) Kützing & + & + & & & + & \\
\hline Gomphonema pseudoaugur Lange-Bertalot & + & + & & & & \\
\hline Gomphonema pumilum (Grunow) E. Reichardt \& Lange-Bertalot & + & + & & + & & + \\
\hline $\begin{array}{l}\text { Gomphonema pumilum var. rigidum E. Reichardt \& Lange- } \\
\text { Bertalot }\end{array}$ & & + & & & & \\
\hline Gomphonema rhombicum Fricke & & & & & + & \\
\hline $\begin{array}{l}\text { Gomphonema saprophilum (Lange-Bertalot \& E. Reichardt) } \\
\text { Abraca et al. }\end{array}$ & + & & & & & \\
\hline Gomphonema subclavatum (Grunow) Grunow & & + & & & & \\
\hline Gomphonema tergestinum (Grunow) Fricke & & & & & & + \\
\hline Gomphonema truncatum Ehrenberg & & & + & & + & \\
\hline Gomphonema sp. & & + & & & & \\
\hline Gyrosigma acuminatum (Kützing) Rabenhorst & & & & & + & \\
\hline Gyrosigma attenuatum (Kützing) Rabenhorst & & + & & & & \\
\hline Halamphora coffeiformis (C. Agardh) Levkov & & + & + & + & & + \\
\hline Halamphora veneta (Kützing) Levkov & & + & & + & & + \\
\hline Hantzschia abundans Lange-Bertalot & + & & & & + & \\
\hline Hantzschia amphioxys (Ehrenberg) Grunow & & + & & & & \\
\hline $\begin{array}{l}\text { Hippodonta capitata (Ehrenberg) Lange-Bertalot, Metzeltin \& } \\
\text { Witkowski }\end{array}$ & & + & & & + & \\
\hline $\begin{array}{l}\text { Hippodonta hungarica (Grunow) Lange-Bertalot, Metzeltin \& } \\
\text { Witkowski }\end{array}$ & & & & + & & \\
\hline Lemnicola exigua (Grunow) Kulikovskiy, Witkowski \& Plinski & + & & & & + & \\
\hline Lemnicola hungarica (Grunow) Round \& Basson & & + & & & & \\
\hline
\end{tabular}




\begin{tabular}{|c|c|c|c|c|c|c|}
\hline \multirow[t]{2}{*}{ Taxa } & \multicolumn{2}{|c|}{ Stream } & \multicolumn{2}{|c|}{ Lake } & \multicolumn{2}{|c|}{ Reservoir } \\
\hline & Sp. & Aut. & Sp. & Aut. & Sp. & Aut. \\
\hline Lindavia balatonis (Pantocsek) Nakov et al. & & & & & + & + \\
\hline Luticola ventricosa (Kützing) D.G. Mann & + & & & & + & \\
\hline Mastogloia elliptica (C. Agardh) Cleve & & + & & & & \\
\hline Mastogloia smithii Thwaites ex W. Smith & + & + & + & + & & \\
\hline Mastogloia cf. pseudosmithii Lee et al. & & & + & & & \\
\hline Melosira varians C. Agardh & & + & & & + & \\
\hline Navicymbula pusilla (Grunow) Krammer & + & + & + & + & + & + \\
\hline Navicula antonii Lange-Bertalot & + & + & + & + & + & + \\
\hline Navicula capitatoradiata $\mathrm{H}$. Germain & + & + & + & + & + & + \\
\hline Navicula cari Ehrenberg & & + & & & & + \\
\hline Navicula cincta (Ehrenberg) Ralfs & & + & + & & + & \\
\hline Navicula cryptocephala Kützing & + & + & & & & + \\
\hline Navicula cryptotenella Lange-Bertalot & + & + & + & + & & + \\
\hline Navicula digitoconvergens Lange-Bertalot & & & & + & & \\
\hline Navicula erifuga Lange-Bertalot & + & & & & & \\
\hline Navicula gottlandica Grunow & & & & & + & \\
\hline Navicula gregaria Donkin & & & & & & + \\
\hline Navicula hanseatica Lange-Bertalot \& Stachura & & & & & + & \\
\hline Navicula lanceolata Ehrenberg & + & & & & + & \\
\hline Navicula menisculus Schumann & + & & & & + & + \\
\hline Navicula metareichardtiana Lange-Bertalot \& Kusber & + & + & & & & \\
\hline Navicula notha J.H. Wallace & + & & & & + & + \\
\hline Navicula phyllepta Kützing & & + & & & & \\
\hline Navicula rhynchotella Lange-Bertalot & + & & & & & \\
\hline Navicula rostellata Kützing & & & & & + & \\
\hline Navicula simulata Manguin & & + & + & & & \\
\hline Navicula striolata (Grunow) Lange-Bertalot & & & + & & & \\
\hline Navicula tripunctata (O.F. Müller) Bory & + & + & & & & + \\
\hline Navicula trivialis Lange-Bertalot & & & & & & + \\
\hline Navicula upsaliensis (Grunow) M. Peragallo & & & + & & & \\
\hline Navicula vandamii Schoeman \& R.E.M. Archibald & & + & & & & \\
\hline Navicula veneta Kützing & + & + & & & & \\
\hline
\end{tabular}




\begin{tabular}{|c|c|c|c|c|c|c|}
\hline \multirow[t]{2}{*}{ Taxa } & \multicolumn{2}{|c|}{ Stream } & \multicolumn{2}{|c|}{ Lake } & \multicolumn{2}{|c|}{ Reservoir } \\
\hline & Sp. & Aut. & Sp. & Aut. & Sp. & Aut. \\
\hline Navicula viridula var. germainii (Wallace) Lange-Bertalot & & + & & + & & + \\
\hline Navicula sp. & & & & + & & \\
\hline Neidium affine (Ehrenberg) Pfitzer & & & & & & + \\
\hline Nitzschia alpina Hustedt & + & + & & & + & + \\
\hline Nitzschia amphibia Grunow & + & + & + & & + & \\
\hline Nitzschia bulnheimiana (Rabenhorst) H.L. Smith* & & + & & & & \\
\hline Nitzschia capitellata Hustedt & + & + & & & & + \\
\hline Nitzschia denticula Grunow & + & + & & & + & + \\
\hline Nitzschia desertorum Hustedt & & & & + & & \\
\hline Nitzschia dissipata (Kützing) Rabenhorst & + & + & + & & + & + \\
\hline Nitzschia filiformis (W. Smith) Van Heurck & & + & & & & \\
\hline Nitzschia fonticola (Grunow) Grunow & + & & & + & & + \\
\hline Nitzschia frustulum (Kützing) Grunow & + & + & + & + & & + \\
\hline Nitzschia gracilis Hantzsch & & & & + & & \\
\hline Nitzschia hantzschiana Rabenhorst & + & + & & & + & \\
\hline Nitzschia heufleriana Grunow & + & & & & + & \\
\hline Nitzschia inconspicua Grunow & + & + & + & + & & + \\
\hline Nitzschia linearis W. Smith & + & + & + & & + & \\
\hline Nitzschia palea (Kützing) W. Smith & + & + & & + & + & + \\
\hline Nitzschia palea var. debilis (Kützing) Grunow & & + & & & & + \\
\hline Nitzschia palea var. minuta (Bleisch) Grunow & + & & & & & \\
\hline Nitzschia pusilla Grunow & + & & & + & & \\
\hline Nitzschia recta Hantzsch ex Rabenhorst & & & & & + & \\
\hline Nitzschia rosenstockii Lange-Bertalotii & + & & & & & \\
\hline Nitzschia sociabilis Hustedt & & + & & & & + \\
\hline Nitzschia solita Hustedt & + & & & + & & \\
\hline Nitzschia supralitorea Lange-Bertalot & & + & & & & + \\
\hline Nitzschia tenuis W. Smith & + & & & & & \\
\hline Nitzschia tubicola Grunow & & + & & & & \\
\hline Nitzschia umbonata (Ehrenberg) Lange-Bertalot & & + & & & & \\
\hline Pantocsekiella iranica (Nejdsattari et al.) Kiss et al. & & + & & + & & + \\
\hline Pantocsekiella ocellata (Pantocsek) Kiss \& Ács & + & + & & & + & + \\
\hline
\end{tabular}




\begin{tabular}{|c|c|c|c|c|c|c|}
\hline \multirow[t]{2}{*}{ Taxa } & \multicolumn{2}{|c|}{ Stream } & \multicolumn{2}{|c|}{ Lake } & \multicolumn{2}{|c|}{ Reservoir } \\
\hline & Sp. & Aut. & Sp. & Aut. & Sp. & Aut. \\
\hline Paraplaconeis minor (Grunow) Lange-Bertalot* & & & & & + & \\
\hline $\begin{array}{l}\text { Paraplaconeis placentula (Ehrenberg) Kulikovskiy \& Lange- } \\
\text { Bertalot }\end{array}$ & + & & & + & & \\
\hline Pinnularia brebissonii (Kützing) Rabenhorst & + & + & & & + & \\
\hline Pinnularia divergens W. Smith & & & & & + & + \\
\hline Pinnularia suchlandtii Hustedt & & & + & & + & \\
\hline Pinnularia sp. & & + & & & & \\
\hline Placoneis anglophila (Lange-Bertalot) Lange-Bertalot* & & & & & + & \\
\hline Placoneis clementis (Grunow) E.J. Cox & & & & & & + \\
\hline Placoneis clementioides (Hustedt) E.J. Cox * & & & & & + & \\
\hline Placoneis ignorata (Schimanski) Lange-Bertalot & & & & & & + \\
\hline Placoneis sp. & & & & & & + \\
\hline Planothidium frequentissimum (Lange-Bertalot) Lange-Bertalot & + & + & + & + & & \\
\hline $\begin{array}{l}\text { Planothidium lanceolatum (Brébisson ex Kützing) Lange- } \\
\text { Bertalot }\end{array}$ & & + & & & & \\
\hline Planothidium rostratum (Østrup) Lange-Bertalot & + & & & & & \\
\hline Pseudofallacia monoculata (Hustedt) Liu, Kociolek \& Wang & + & & & & & \\
\hline $\begin{array}{l}\text { Pseudostaurosira brevistriata (Grunow) D.M. Williams \& } \\
\text { Round }\end{array}$ & + & + & & & & \\
\hline Reimeria sinuata (W. Gregory) Kociolek \& Stoermer & & & & & & + \\
\hline Rhopalodia gibberula (Ehrenberg) Otto Müller & & + & & + & & + \\
\hline Sellaphora absoluta (Hustedt) Wetzel et al. & & & & & & + \\
\hline Sellaphora pupula (Kützing) Mereschkovsky & + & + & + & + & + & + \\
\hline Sellaphora sp. & & & & + & & \\
\hline Stauroneis acidoclinata Lange-Bertalot \& Werum* & & & & + & + & \\
\hline Stauroneis gracilis Ehrenberg & & & + & & & \\
\hline Staurosira dubia Grunow & & & & & + & \\
\hline Staurosira venter (Ehrenberg) Cleve \& J.D. Möller & & & & + & & \\
\hline Staurosirella pinnata (Ehrenberg) D.M. Williams \& Round & & + & & & & \\
\hline Stephanodiscus astraea (Kützing) Grunow & & & + & & + & \\
\hline Surirella amphioxys W. Smith & & & & & & + \\
\hline Surirella angusta Kützing & & + & & & & + \\
\hline Surirella brebissonii Krammer \& Lange-Bertalot & + & + & & & & \\
\hline
\end{tabular}




\begin{tabular}{|c|c|c|c|c|c|c|}
\hline \multirow[t]{2}{*}{ Taxa } & \multicolumn{2}{|c|}{ Stream } & \multicolumn{2}{|c|}{ Lake } & \multicolumn{2}{|c|}{ Reservoir } \\
\hline & Sp. & Aut. & Sp. & Aut. & Sp. & Aut. \\
\hline Surirella librile (Ehrenberg) Ehrenberg & & + & & & + & \\
\hline Surirella minuta Brébisson ex Kützing & + & + & & & & \\
\hline Surirella ovalis Brébisson & + & + & & & & \\
\hline Surirella robusta Ehrenberg & + & & & & & + \\
\hline Surirella subsalsa W. Smith & + & & & & & \\
\hline Tabularia fasciculata (C. Agardh) D.M. Williams \& Round & + & & & & & \\
\hline Tryblionella angustata W. Smith & + & + & & & & \\
\hline Tryblionella apiculata W. Gregory & + & + & + & + & & \\
\hline $\begin{array}{l}\text { Tryblionella brunoi (Lange-Bertalot) Cantonati \& Lange- } \\
\text { Bertalot }\end{array}$ & & & & & & + \\
\hline Tryblionella calida (Grunow) D.G. Mann & & + & & & & \\
\hline Tryblionella hungarica (Grunow) Frenguelli & + & + & & & & \\
\hline Ulnaria acus (Kützing) Aboal & + & + & & & + & + \\
\hline Ulnaria biceps (Kützing) Compère & + & + & & & + & \\
\hline Ulnaria delicatissima (W. Smith) Aboal \& P.C. Silva & & + & & & + & + \\
\hline Ulnaria ulna (Nitzsch) Compère & + & + & & & + & \\
\hline
\end{tabular}

Note. $S p=$ Spring, $A u t=$ Autumn, ${ }^{*}=$ New records.

Amongst the diatom genera, Navicula Bory and Nitzschia Hassall were represented with the highest numbers of taxa (27) in the river basin, this was followed by Gomphonema Agardh (22), Encyonema Kützing (10) and Cymbella Agardh (9). 20 genera were represented with only one species. 136 species were identified in spring, while the number of species increased to 174 in autumn. The species number varied between the environments; 131 taxa were observed in reservoir samples, while 61 and 160 taxa found in lake and stream samples, respectively. The taxa numbers also showed a variance in different habitats between spring and autumn. Total 78 taxa found in spring whereas 86 taxa were detected in autumn in the reservoirs. There were 34 taxa in spring with an increase to 45 taxa in autumn in lakes, and the highest species diversity was found in streams, 90 and 124 taxa in spring and autumn, respectively.

The most abundant species was Navicula antonii (17.1\%) in the whole basin. The other common taxa ( $\geq 5 \%)$ observed were Nitzschia palea var. debilis (15.5\%), Tabularia fasciculata (6.6\%) Cyclotella meneghiniana (5.6\%), Nitzschia frustulum (5.4\%), and Amphora pediculus (5.3\%). The remaining numbers of species showed less than $5 \%$ occurrences in the samples (Figure 2;3;4). 
The most abundant species in the studied areas differed significantly according to the sampling period (Table 4). The number of species which exceeded 5\% was higher in spring than in autumn. Diatom material observed in the reservoir samples showed that Pantocsekiella iranica (in autumn), P. ocellata and Ulnaria delicatissima (in spring) were the most abundant species (14\%, 17\% and $17 \%$, respectively). In the lake samples, abundant species changed and Achnanthidium minutissimum var. jackii (in autumn) and Encyonema caespitosum (in spring) were the most abundant taxa ( $20 \%$ and $35 \%)$. On the other hand, especially in autumn, only two species, Nitzschia palea var. debilis (38\%) and Navicula antonii (38\%), were dominant in the stream samples and other species remained below total RA 5\%.

Table 4

Species Identified Above 5\% of the Total RA in Reservoirs, Lakes And Streams

\begin{tabular}{|c|c|c|c|c|}
\hline & Spring $(\%)$ & & Autumn (\%) & \\
\hline \multirow{5}{*}{ 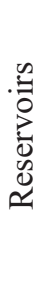 } & Pantocsekiella ocellata & 17 & Pantocsekiella iranica & 14 \\
\hline & Ulnaria delicatissima & 17 & Encyonema lacustre & 9 \\
\hline & Cymbella cymbiformis & 13 & Nitzschia palea & 6 \\
\hline & Aulacoseira ambigua & 10 & Amphora pediculus & 5 \\
\hline & Encyonema ventricosum & 5 & & \\
\hline \multirow{6}{*}{ 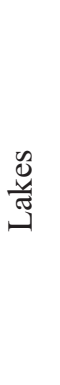 } & Encyonema caespitosum & 35 & $\begin{array}{l}\text { Achnanthidium } \\
\text { minutissimum var. jackii }\end{array}$ & 20 \\
\hline & Fragilaria vaucheriae & 19 & Encyonopsis subminuta & 17 \\
\hline & Navicymbula pusilla & 19 & Gomphonema calcareum & 11 \\
\hline & Navicula capitatoradiata & 9 & Nitzschia fonticola & 7 \\
\hline & & & Mastogloia smithii & 6 \\
\hline & & & Berkeleya sp. & 8 \\
\hline \multirow{6}{*}{ 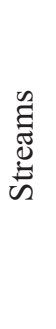 } & Tabularia fasciculata & 15 & Nitzschia palea var. debilis & 38 \\
\hline & Cyclotella meneghiniana & 13 & Navicula antonii & 38 \\
\hline & Amphora pediculus & 11 & & \\
\hline & Nitzschia frustulum & 11 & & \\
\hline & Nitzschia dissipata & 7 & & \\
\hline & Nitzschia palea & 7 & & \\
\hline
\end{tabular}




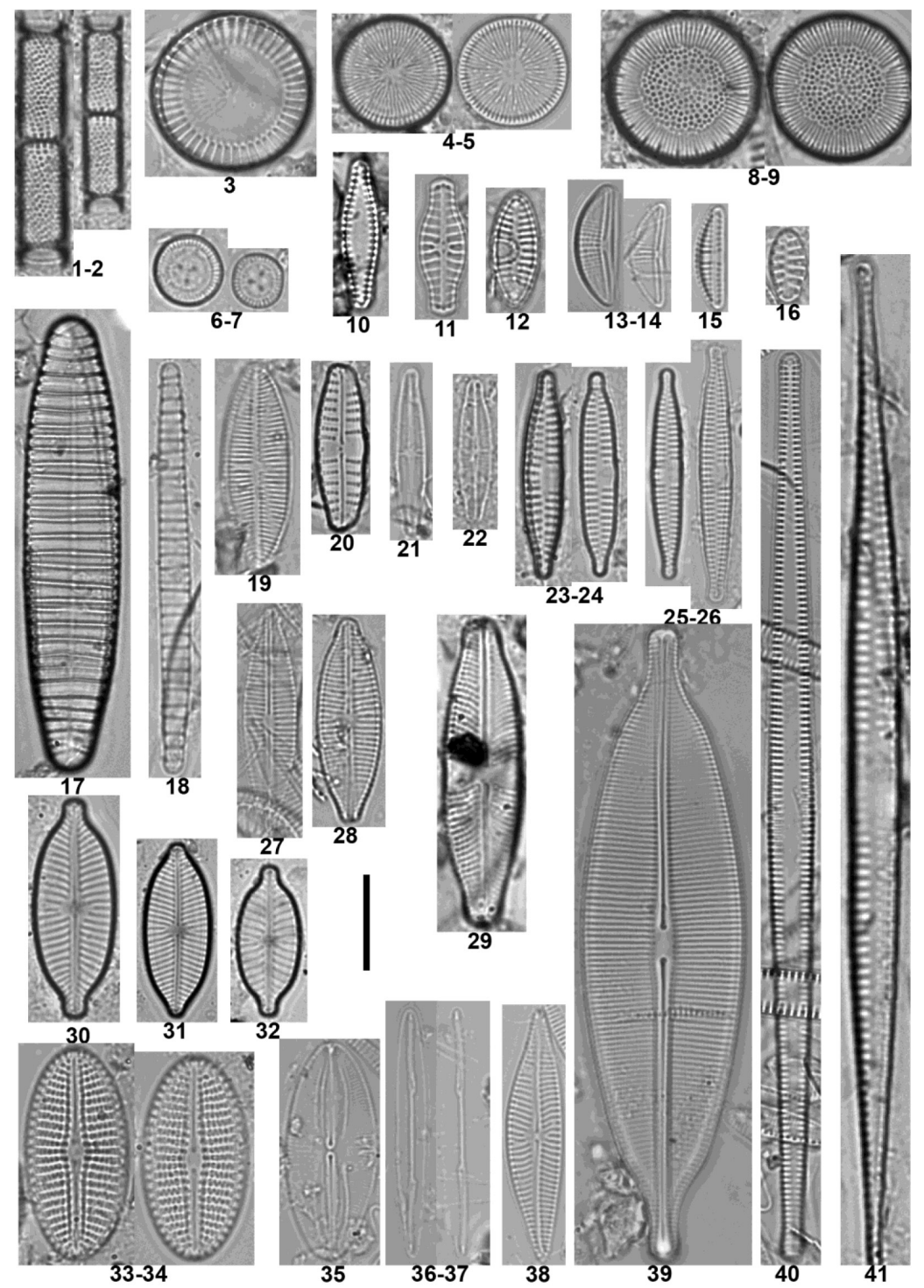

Figure 2. Common Diatoms of Burdur River Basin 1-2. Aulacoseira ambigua; 3. Cyclotella meneghiniana; 4-5. Pantocsekiella iranica; 6-7. P. oceallata; 8-9. Lindavia balatonis; 10. Pseudostaurosira brevistriata; 11. Hippodonta capitata; 12. Planothidium frequentissimum; 13-14. Halamphora veneta; 15. Amphora pediculus; 16. Staurosirella pinnata; 17. Diatoma vulgaris; 18. D. moniliformis; 19. Lemnicola hungarica; 20. Reimeria sinuata; 21. Achnanthidium minutissimum; 22. A. minutissimum var. jackii; 23-24. Fragilaria vaucheriae; 25-26. F. radians; 27. Craticula buderi; 28. C. accomoda; 29. Stauroneis acidoclinata; 30. Placoneis clementoides; 31. Paraplaconeis minor; 32. Placoneis anglophila; 33-34. Diploneis parma; 35. Fallacia pygamea; 36-37. Berkeleya sp.; 38. Navicymbula pusilla; 39. Craticula ambigua; 40. Tabularia fasciculata; 41. Ulnaria acus. Scale bar: $10 \mu \mathrm{m}$. 

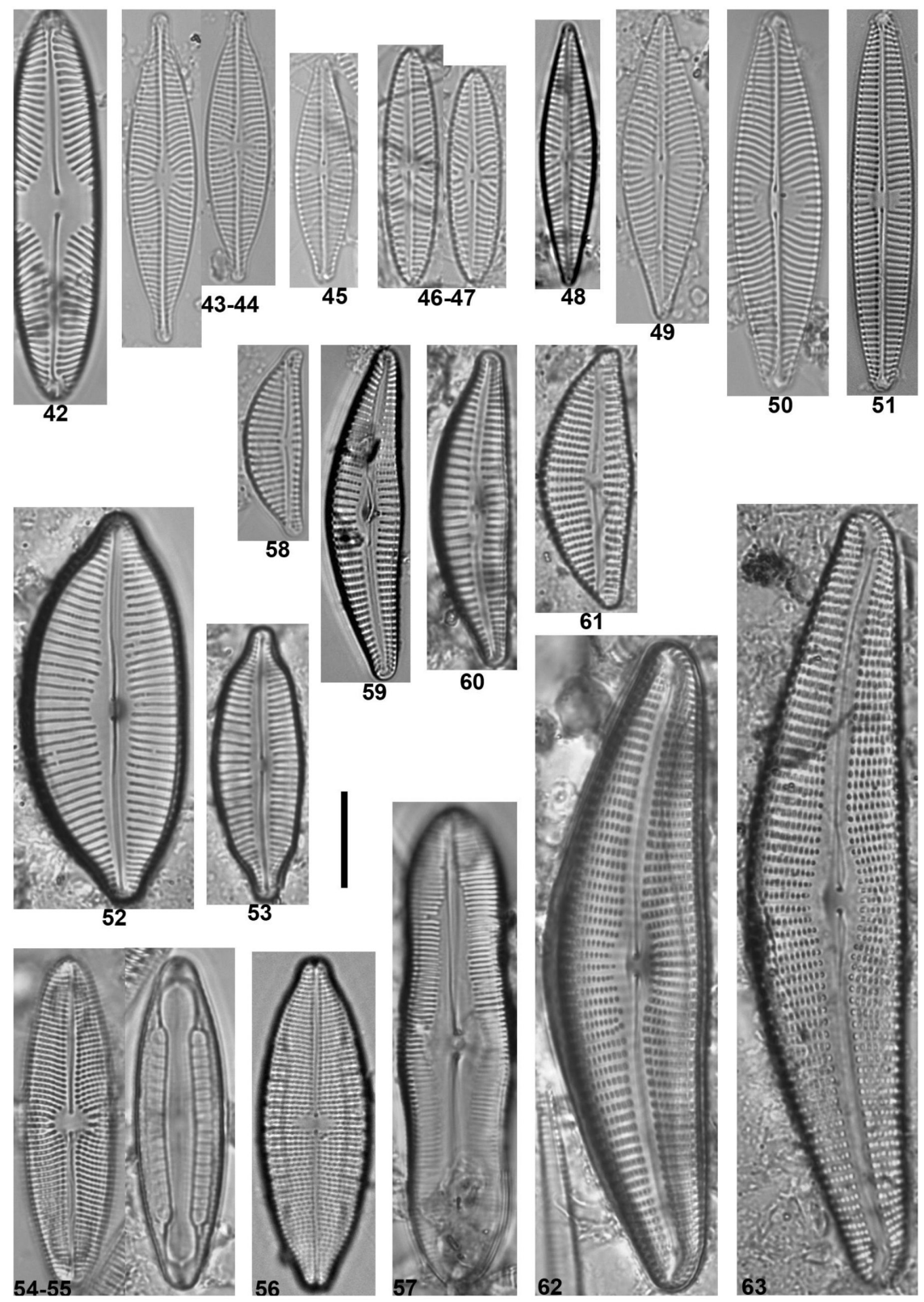

Figure 3. 42. Pinnularia brebissonii; 43-44. Navicula capitatoradiata; 45. N. veneta; 4647. $N$. cincta; 48. N. cryptotenella; 49. N. trivialis; 50. N. exilis; 51. N. tripunctata; 52. Cymbopleura amphicephala; 53. C. lata; 54-55. Mastogloia elliptica; 56. M. smithii; 57. Pinnularia silicula; 58. Encyonema ventricosum; 59. E. vulgare; 60. Cymbella affinis; 61. Encyonema caespitosum; 62. Cymbella lange-bertalotii; 63. C. cymbiformis. Scale bar: 10 $\mu \mathrm{m}$. 

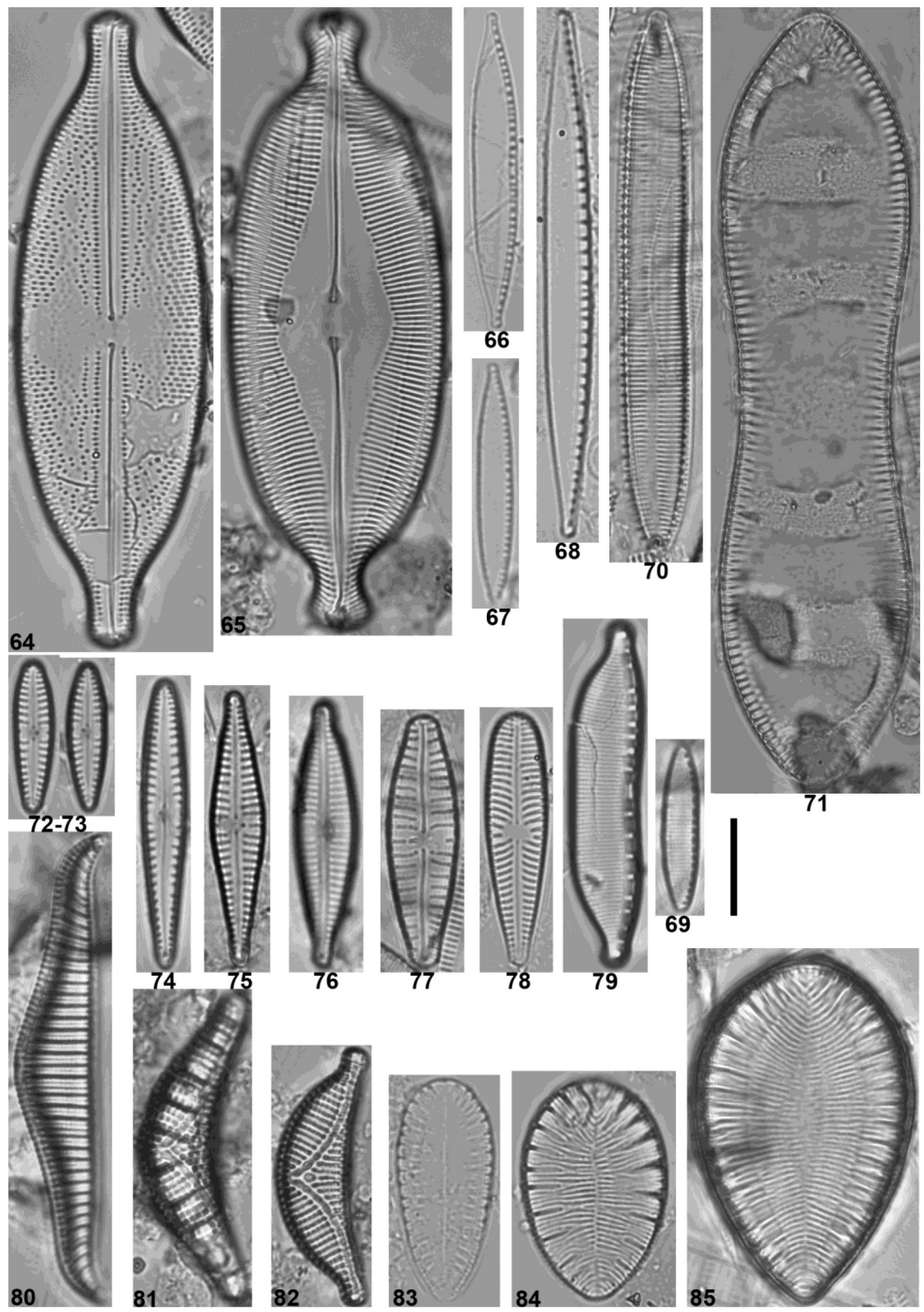

Figure. 4. 64. Anomoeoneis sphaerophora; 65. Caloneis amphisbaeana; 66. Nitzschia palea; 67. N. palea var. debilis; 68. N. recta; 69. N. bulnheimiana; 70. Tryblionella hungarica; 71. Surirella librile; 72-73. Gomphonema pumilum var. rigidum; 74. G. pumilum; 75. G. auritum; 76. G. exilissimum; 77. G. drutelingense; 78. G. olivaceum. 79. Hantzschia amphioxys; 80. Epithemia gibba; 81. E. smithii; 82. E. sorex; 83. Surirella minuta; 84. S. brebissonii; 85. S. ovalis. Scale bar: $10 \mu \mathrm{m}$. 


\section{Discussion and Conclusion}

Burdur River Basin is one of the two smallest basins out of 25 in Turkey. In this research, diatom composition and its distribution through the streams, lakes and reservoirs of the basin were studied for the first time in detail, and the diversity was found relatively higher in comparison with different river basins. Solak et al. (2018) found 94 taxa from Küçük Menderes River Basin, Çelekli et al. (2018) reported 80 taxa from the North Aegean catchment while 148 taxa reported from western Black Sea River catchment (Özer et al., 2018) and Demir et al. (2017) observed 64 diatom taxa from Akarçay River Basin. Similarly, 65 diatom taxa were found in Aras River catchment (Çelekli et al., 2019). The highest number of taxa observed in Burdur area could be the result of the variation of physico-chemical and geological characteristics of the aquatic ecosystems in the basin. The lower diversity was also detected in several diatom composition studies conducted in some lakes and rivers, Çiçek \& Yamuç (2017) found out 42 diatom taxa in Eğirdir Lake, Isparta Province and Karacaoğlu \& Dalkıran, (2017) detected 134 taxa in Nilüfer Stream and Şanal \& Demir (2018) studied epiphytic samples of Lake Mogan and 58 diatoms species were observed. Since the samples were taken seasonally or even monthly in these studies, a more diverse diatom community was typically expected in comparison to the present study. However, focusing on one type of ecosystem (lake or river) and relatively similar characteristics of sampling points compared to whole basin studies could be the reason for detecting lower diversity of diatoms.

Diatom diversity of Burdur River Basin varied among streams (160 taxa), lakes (61 taxa) and reservoirs (131 taxa). Besides, the diatom community structure also differed among the habitats (Table 4). Pantocsekiella ocellata, P. iranica and Ulnaria delicatissima were dominant species in the reservoirs, but did not observe as dominant species in other habitats.

The seasonal changes of dominant taxa were also remarkable in the same habitat. The most distinct variation was in streams. Dominant taxa number was six in spring and declined to two species in autumn when Navicula antonii (37.7\%) and Nitzschia palea var. debilis (37.8 \%) were dominant and constituted $74.4 \%$ of total abundance.

Navicula Bory and Nitzschia Hassall are generally the most diverse and widespread genera in freshwater diatoms (Karacaoğlu \& Dalkıran, 2017; Kociolek et al. 2019). In Burdur River Basin, the results are consisted with this general trend and Navicula and Nitzschia species diversity were high; however, their relative 
abundances were low. Aside from the dominant taxa, Navicula antonii and Nitzschia palea var. debilis, only Navicula capitatoradiata, Nitzschia dissipata, N. frustulum and $N$. palea were represented over $5 \%$.

Diatoms are essential tools for bio-assessment of aquatic ecosystems and the identification of the taxa together with its ecological requirements would contribute further to the detection of water quality. Burdur River Basin is a closed basin, which has no connection to the sea and salinity gradient of the habitats were very broad. Therefore, the species composition found in the basin comprised the species with different tolerances to the salinity. Some species prefer waters with high salinity, while others may have a wide salinity tolerance (Schröder et al., 2015). In the present study, we detected Navicula antonii and Nitzschia frustulum in all habitats with different salinity. Although $N$. antonii and $N$. frustulum species are generally defined as freshwater species, they have also been identified in Ebro Estuary which is a salt wedge estuary in the Mediterranean (Rovira et al., 2009; Costa-Böddeker et al., 2017). Our results confirmed their euryhaline characteristics based on their presence in the basin from fresh to saline habitats.

Two unique habitats related to salinity in the basin are Burdur and Acıgöl Lakes. Our observations indicated that some taxa which are present in brackish and marine waters found in these sampling areas. Specifically, the brackish species, Halamphora coffeiformis and Navicula cincta were dominant in Acıgöl Lake and their relative abundance was $69 \%$ for $N$. cincta in spring and $40 \%$ for $H$. coffeiformis in autumn. Similarly, species such as Berkeleya sp., H. coffeiformis, Navicula simulata, Tryblionella apiculata, which are known to be found in both marine and brackish waters, also showed significant presence in Burdur Lake. Some taxa like Tabularia fasciculata which is common in the marine coastal areas (Baytut and Gönülol, 2016) were observed in the river basin with an accomplice of a high number of taxonomically complex taxa (Navicula, Nitzschia, Tryblionella, Surirella). Species like Halamphora coffeiformis, Navicula capitatoradiata, N. cincta, N. erifuga, N. hanseatica, Nitzschia tubicola, Tryblionella apiculata, T. hungarica were assigned to marine or brackish water ecosystems and also in freshwaters with high electrolyte content (Guiry \& Guiry, 2019). Furthermore, Akbulut (2010) reported N. cincta and T. apiculata in the Tuz Lake basin which is under the brackish, saline category. Species with high tolerance to salinity living in brackish waters or freshwaters with high conductivity could be found in different habitats, like some freshwater taxa observed in the marine coasts. Another taxon which generally referred to as marine species is Berkeleya sp. (Figure 2). This species resembles Berkeleya fennica, a brackish species previously reported from the Baltic Sea (Witkowski et al. 2000) and occurred 
mainly in brackish and a few freshwater habitats of Burdur river basin. Nevertheless, ultrastructure details are needed for the identification of the taxa.

In this research, a total of 223 taxa were found with a 11 new records for Turkey. These taxa were Craticula buderi, Cymbella lange-bertalotii, Encyonema vulgare, Gomphonema auritum, G. drutelingense, G. exilissimum, Nitzschia bulnheimiana, Paraplaconeis minor, Placoneis anglophila, Placoneis clementoides and Stauroneis acidoclinata. The results would contribute to the knowledge of diatom distribution in Turkey and Burdur River Basin, in particular. To determine the ecological quality of the river basins, taxonomical results would be a supplement for the physicochemical parameters for further studies. The results extend the biogeography of diatoms in Turkey and contribute to the knowledge of the diatom composition and distribution in the river basin.

\section{Acknowledgements}

This study was supported by the Ministry of Agriculture and Forestry, General Directorate of Water Management. 


\section{References}

Akbulut, A. (2010). The diatom composition of the Salt Lake Basin and its relationship with salinity. Ekoloji, 159, 150-159. doi: 10.5053/ekoloji.2010.7418

Atıc1, T. (1997). Sakarya River pollution and algae. Ekoloji ve Çevre Dergisi, 6(24), 28 -32 (in Turkish).

Ács, É., Szabó, K., Tóth, B. \& Kiss, K.T. (2004). Investigation of benthic algal communities, especially diatoms of some Hungarian streams in connection with reference conditions of the Water Framework Directives. Acta Botanica Hungarica, 46, 225-278.

Barlas, M., Mumcu, M.F., Solak C.N. \& Çoban, O. (2002). Akçapınar Deresi ve Kadın Azmağı Deresi (Muğla) epilitik alg florasının su kalitesine bağlı olarak incelenmesi. VI. Ulusal Biyoloji Kongresi. 4-7 Eylül 2002, Malatya, pp. 65-68 (in Turkish).

Baytut, Ö., \& Gönülol, A. (2016). Phytoplankton distribution and variation along a freshwater-marine transition zone (Kizllırmak River) in the Black Sea. Oceanological and Hydrobiological Studies, 45(4), 453-465.

Costa-Böddeker, S., Schwarz, A., Huy, H. Đ., \& Schwalb, A. (2017). Diatom assemblages in surface sediments along nutrient and salinity gradients of Thi Vai estuary and Can Gio mangrove forest, Southern Vietnam. Estuaries and coasts, 40(2), 479-492.

Çelekli, A., Toudjani, A.A., Lekesiz, H. \& Çetin, T. (2018). Ecological quality assessment of running waters in the North Aegean Catchment with diatom metrics and multivariate approach. Limnologica, 73, 20-27. https://doi.org/10.1016/j.limno.2018.09.001

Çelekli, A., Kayhan, S., Lekesiz, Ö., Toudjani, A.A. \& Çetin, T. (2019). Limno-ecological assessment of Aras River surface waters in Turkey: application of diatom indices. Enviromental Science and Pollution Research, 26 (8), 8028-8038. https://doi.org/10.1007/s11356-019-04295-y

Çiçek, N. L., \& Yamuç, F. (2017). Using epilithic algae assemblages to assess water quality in Lake Kovada and Kovada Channel (Turkey), and in relation to environmental factors. Turkish Journal of Fisheries and Aquatic Sciences, 17(4), 701-711.

Dalkıran, N., Zünbülgil, B., Karacaoğlu, D., \& Şükran, Dere (2016). Uluabat Gölü Epifitik Diyatomelerinin Uzun Dönemdeki Değişimi. Journal of Limnology and Freshwater Fisheries Research, 2(3), 153-163 (in Turkish).

Demir, N., Çetin, T., Gök, C., \& Şanal, M. (2017). First biological monitoring in the Akarçay Basin according to the water framework directive: Phytoplankton and phytobenthos. Turkish Journal of Water Science \& Management, 1(1), 90-105.

Guiry, M. D., \& Guiry, G. M. (2019). AlgaeBase. World-wide electronic publication, National University of Ireland, Galway. Accessed at http://www.algaebase.org 
Hofmann G., Werum, M., \& Lange-Bertalot, H. (2011). Diatomeen im Süßwasser - Benthos von Mitteleuropa. Bestimmungsflora Kieselalgen für die ökologische Praxis. Über 700 der häufigsten Arten und ihre Ökologie. A.R.G. Gantner Verlag, Rugell. pp.908.

Karacaoğlu, D., \& Dalkıran, N. (2017). Epilithic diatom assemblages and their relationships with environmental Epilithic diatom assemblages and their relationships with environmental variables in the Nilüfer Stream Basin, Bursa, Turkey. Environmental Monitoring Assessment, 189, 1-22.

Kociolek, J.P., Balasubramanian, K., Blanco, S., Coste, M., Ector, L., Liu, Y., ... Witkowski, J. (2019). DiatomBase. Retrieved August 31, 2017, from http://www.diatombase.org

Krammer, K. \& Lange-Bertalot, H. (1986). Bacillariophyceae. 1. Teil: Naviculaceae. Ettl, H., Gerloff, J., Heynig, H. and Mollenhauer, D (Ed.). Süsswasser flora von Mitteleuropa, Band 2/1. Stuttgart: Gustav Fischer Verlag.

Krammer, K., \& Lange-Bertalot, H. (1988). Bacillariophyceae. 2. Teil: Bacillariaceae, Epithemiaceae, Surirellaceae. Ettl, H., Gerloff, J., Heynig, H. and Mollenhauer, D. (Ed.). Süsswasserflora von Mitteleuropa, Band 2/2. Stuttgart: Gustav Fischer Verlag.

Krammer, K., \& Lange-Bertalot, H. (1991a). Bacillariophyceae. 3. Teil: Centrales, Fragilariaceae, Eunotiaceae. Ettl, H., Gerloff, J., Heynig, H. and Mollenhauer, D. (Ed.). Süsswasserflora von Mitteleuropa, Band 2/3. Stuttgart: Gustav Fischer Verlag.

Krammer, K., \& Lange-Bertalot, H. (1991b). Bacillariophyceae. 4. Teil: Achnanthaceae, Kritische Ergänzungen zu Navicula (Lineolatae) und Gomphonema, Gesamtliteraturverze ichnis Teil 1-4. Ettl, H., Gärtner, G., Gerloff, J., Heynig, H. And Mollenhauer, D. (Ed.). Süsswasserflora von Mitteleuropa, Band 2/4. Stuttgart: Gustav Fischer Verlag.

Kulikovskiy, M., Glushchenko, A.M., Kuznetsova, I.V., \& Genkal, S.I. (2016). Identification book of diatoms from Russia. pp. 1-804, 165 pls. Yaroslavl: Filigran. [in Russian].

Özer, T., Erkaya, I.A., Solak, C.N., \& Udoh, A.U. (2018). Diversity and ecology of algae from Melen River (Western Black Sea River Catchment) in Turkey. Turkish Journal of Fisheries and Aquatic Sciences. 18, 1187-1194.

Rimet, F. (2012). Recent views on river pollution and diatoms. Hydrobiologia, 638, 1-24.

Rovira, L., Trobajo, R., \& Ibanez, C. (2009). Periphytic diatom community in a Mediterranean salt wedge estuary: the Ebro Estuary (NE Iberian Peninsula). Acta Botanica Croatica, 68(2), 285-300.

Schröder, M., Sondermann, M., Sures, B., \& Hering, D. (2015). Effects of salinity gradients on benthic invertebrate and diatom communities in a German lowland river. Ecological indicators, 57, 236248.

Solak, C.N., Çetin, T., Kaleli, A. (2018). Distribution of benthic diatom (phytobenthos) composition in Küçük Menderes River Basin. Turkish Journal of Water Science \& Management, 2, 2-35. 
Solak, C.N., Çetin, T., Karaaslan, Y., Kaleli, A., Yılmaz, E., Duran, M., Kıvanç, G., Kirmençe, T., Koyunluoğlu, Ş.A., Çankaya, F., Y1lmaz, D. (2019). Common Diatoms of Phytobenthos in Gediz River Basin. Turkish Journal of Water Science \&Management, 3(2), 58-70.

Şanal, M., \& Demir, N. (2018). Use of the epiphytic diatoms to estimate the ecological status of Lake Mogan. Applied Ecology and Environmental Research. 16(3), 3529-3543.

Turkish Official Gazette. (2019). Biyolojik İzleme Tebliği ( Publication No: 30808) Retrieved from https://www.resmigazete.gov.tr/eskiler/2019/06/20190621.pdf

Water Framework Directive. (2000). Common implementation strategy for the water framework directive (2000/60/EC).

Witkowski, A., Lange-Bertalot, H., \& Metzeltin, D. (2000) Diatom Flora of Marine Coasts I. In: Iconographia Diatomologica 7. Konigstein: Koeltz Scientific Books. 


\section{Extendend Turkish Abstract \\ (Genişletilmiş Türkçe Özet)}

\section{Burdur Nehir Havzasındaki Farklı Habitatlarda Bentik Diyatome Kompozisyonu ve Dağılımı}

Diyatomeler, sucul sistemlerdeki biyolojik izleme çalışmalarında önemli bir bileşendir. Avrupa Birliği Su Çerçeve Direktifi'nin (2000/60/AT) (SÇD) yürürlüğe girmesini izleyen yıllarda, uyum süreçleri kapsamında Türkiye'de de havza bazında izleme çalışmaları başlamıştır. Bu çerçevede, Türkiye'deki 25 havza içerisinde en küçük alana sahip iki havzadan biri olan Burdur Havzası'nın bentik diyatome kompozisyonu ilk defa bu çalışma ile detaylı olarak incelenmiştir.

Fitobentoz (diyatome) örneklemesi Nisan ve Ekim 2018'de iki dönemde gerçekleştirilmiş, fizikokimyasal parametreler ise Ocak-Aralık 2018 döneminde aylık olarak izlenmiştir. Havzada örnekleme noktası olarak belirlenen toplam 30 istasyonun 7'sinin her iki örnekleme döneminde kuru olduğunun tespit edilmesi nedeniyle 13'ü akarsu, 4'ü doğal göl ve 6's1 rezervuar olmak üzere toplamda 23 istasyonda örnekleme çalışması gerçekleştirilmiştir. 21.06.2019 tarihli Resmi Gazete'de yayınlanan Biyolojik İzleme Tebliği uyarınca fitobentoz örneklemesinde ağırlıklı olarak nehirlerde epilitik alg örneklemesi, göl ve rezervuarlarda ise epifitik alg örneklemesi yapılması gerekmektedir. Ancak, özellikle su seviyesindeki değişimler ve suyun fizikokimyasal özelliklerine bağlı olarak makrofit tespit edilemeyen göl ve rezervuarlarda ikinci sırada önerilen epilitik algler örneklenmiştir. Alınan örnekler $\% 10 \mathrm{HC} 1$ ile muamele edilmiş sonrasında $\mathrm{H}_{2} \mathrm{O}_{2}$ ile yakılarak organik maddelerin uzaklaştırılması sağlanmıştır. Diyatome örnekleri Naphrax ${ }^{\circledR}$ kullanılarak sabit preparatlar haline getirilmiştir. Sayım ve teşhisler için Zeiss Axio Observer Z1 (Carl Zeiss mikroskobu Gmbh, Jena, Almanya) mikroskobu kullanılmıştır. Fitobentoz türlerinin teşhisinde Krammer ve Lange-Bertalot (1986; 1988; $1991 \mathrm{a}, \mathrm{b})$, Hofmann ve ark., (2011), Kulikovskiy ve ark., (2016), Guiry ve Guiry (2019) ve Kociolek ve ark., (2019) kaynaklarından yararlanılmıştır. Her bir örnekten en az 300 diyatome frustülü sayılarak, türlerin nispi bolluğu, sayılan toplam frustüllerin yüzdesi olarak ifade edilmiştir (\% cinsinden nispi bolluk).

Fizikokimyasal ölçümler, havzada yer alan göl, rezervuar ve nehirlerin genel olarak alkali yapıda olduğunu ortaya koymuştur. Ortalama elektriksel iletkenlik (EI) genel olarak rezervuar $(0,5$ $\left.\mathrm{mS} \mathrm{cm}{ }^{-1}\right)$ ve nehirlerde $\left(1,7 \mathrm{mS} \mathrm{cm}^{-1}\right)$ düşük tespit edilirken göllerde $\left(22.3 \mathrm{mS} \mathrm{cm} \mathrm{c}^{-1}\right)$ nispeten daha yüksek ölçülmüştür. Özellikle doğal göller içerisinde yer alan Acıgöl $\left(57.2 \mathrm{mS} \mathrm{cm}^{-1}\right)$ ve Burdur $(29.4$ $\mathrm{mS} \mathrm{cm}{ }^{-1}$ ) göllerinin Eİ değerlerinin yıl boyunca diğer örnekleme noktalarına göre oldukça yüksek olduğu görülmüştür. Y1l boyunca tüm istasyonlarda çözünmüş oksijen (ÇO) değerlerinde önemli değişiklikler gözlemlenmiştir. Burdur ve Acıgöl'de zaman zaman çok düşük ÇO değerleri gözlense de (sırasıyla, 2,7 $\mathrm{mg} \mathrm{L}^{-1}$; $1 \mathrm{mg} \mathrm{L}^{-1}$ ) ortalama değerler Burdur Gölü için $6,05 \mathrm{mg} \mathrm{L}^{-1}$, Acıgöl için ise $7,75 \mathrm{mg} \mathrm{L}^{-1}$ olarak tespit edilmiştir. Havzada çalışılan 13 akarsu, 4 göl ve 6 baraj gölünde toplam 223 takson gözlenmiştir. İlkbaharda 136 tür tespit edilirken, sonbaharda tür çeşitliliği 174 tür olarak tespit edilmiştir. Cins seviyesinde en çok tür çeşitliliği 27 adet tür ile Navicula ve Nitzschia cinslerinde olup bunu 22 adet tür ile Gomphonema cinsi izlemiştir. Navicula antonii ve Nitzschia palea var. debilis türleri 17,1 \% ve 15,5\% ile en yaygın türler olarak tespit edilmiştir. Craticula buderi, Cymbella langebertalotti, Encyonema vulgare, Gomphoenma auritum, G. drutelingense, G. exilissimum, Nitzschia bulnheimiana, Paraplaconeis minor, Placoneis anglophila, Placoneis clementoides ve Stauroneis acidoclinata olmak üzere toplamda 11 tür Türkiye sularında ilk kez gözlenmiştir. Rezervuar, göl ve akarsularda tespit edilen tür sayıları farklılık göstermiş ve en yüksek tür çeşitliliğine akarsularda rastlanılmıştır. Çeşitliliğin akarsularda 160 tür ve rezervuarlarda 131 tür arasında değiştiği görülmüş, 
bununla birlikte, göllerde biyolojik çeşitlilik daha düşük bulunmuştur (61 tür). Rezervuar, göl ve akarsu istasyonlarında tespit edilen türlerin kendi içlerinde nispi bollukları hesaplanmış ve toplam nispi bolluğun $\% 5$ ve üzerini oluşturan türler ayrıca değerlendirilmiştir. Buna göre, rezervuar örneklerinde Pantocsekiella iranica (\%14) ve P. ocellata (\%17) ve Ulnaria delicatissima (\%17) en bol bulunan türler olurken, göl örneklerinde bu türlerin yerini Achnanthidium minutissimum var. jackii (sonbaharda \%20) ve Encyonema caespitosum (ilkbaharda \%35) almıştır. Akarsu örneklerinde ise ilkbaharda Tabularia fasciculata (\%15) en bol bulunan tür olurken sonbaharda Nitzschia palea var. debilis (\%38) ve Navicula antonii (\%38) dışında toplam nispi bolluğun \%5'inin üzerine çıkan türün olmadığı görülmüştür. Navicula ve Nitzschia cinslerine ait türler tatlı su habitatlarında yüksek çeşitliliğe sahip ve yaygın olarak bulunan cinslerdir. Burdur Nehir Havza'sında da bu cinslere ait tür çeşitliliği yüksek bulunsa da, $N$ antonii ve $N$. palea var. debilis türleri dışında toplam nispi bollukları düşük bulunmuştur.

Burdur Nehir Havzası, denizle bağlantısı olmayan kapalı bir havzadır ve Burdur ve Acıgöl gibi yüksek tuzluluk karakterindeki göllere sahiptir. Bu çalışma sonucunda, havzada acısu ve deniz kıyı bölgelerinde yayılış gösterdiği bilinen bazı türler tespit edilmiştir. Özellikle Acıgöl'de acısularda bulundukları bilinen Halamphora coffeiformis ve Navicula cincta türleri oldukça yüksek sayılara ulaşmıştır. Benzer şekilde, hem deniz hem de acı sularda bulunduğu bilinen Berkeleya sp., Halamphora coffeiformis, Navicula simulata, Tryblionella apiculata gibi türlerin Burdur Gölü'nde önemli miktarda varlık gösterdiği tespit edilmiştir. Havzada tespit edilen, Navicula capitatoradiata, N. erifuga, $N$. hanseatica, Nitzschia tubicola, Tryblionella apiculata, Tabularia fasciculata gibi türlerin ekolojik tercihleri açısından deniz ve acısularda bulunduklarına dair kayıtlar mevcuttur (Guiry \& Guiry, 2019). Navicula antonii türü ise havzada farklı tuzluluk seviyelerine sahip tüm alanlarda bulunmuştur. $\mathrm{Bu}$ durum türün örihalin bir tür olduğunu göstermektedir.

Burdur Nehir Havzası'nda yapılan bu çalışma bölgedeki ilk detaylı çalışmadır ve Türkiye diyatome florasına 11 yeni kayıt türün ilave edilmesini sağlamıştır. Burdur Havzası'nda gözlenenen yüksek biyoçeşitlilik havza içi sucul ekosistemlerin çeşitliliğinin bir sonucudur. Ayrıca, bu çalışmada kapalı bir havza olan Burdur Havzası'nın diğer havzalardan daha farklı diyatome kompozisyonuna sahip olduğu gözlenmiştir. Elde edilen veriler, Burdur Havzası benzeri özel alanlarda yapılacak izleme çalışmalarında önemli olacaktır. 